\title{
Research on the Application of Internet Technology in Real Life Practice
}

\author{
Miao Zhuang
}

Xi'an International University, school of information and network, Xi'an, Shaanxi ,China

\author{
Keywords: Application, Internet Technology, Real Life Practice
}

\begin{abstract}
Internet Technology is seen as an extension of the application of the Internet, it is known as the third revolutionary innovation of information technology. Application innovation is the core of the development of Internet Technology, the innovation which taking the user experience as the core is the soul of the development of things. This paper mainly introduces the characteristics of Internet Technology, technology and the application of Internet Technology, and expounds the application and development of Internet Technology and other related issues.
\end{abstract}

\section{Introduction}

Throughout the development and management of all walks of life around the world, network information technology is almost everywhere, and the integration of radio frequency identification technology, laser scanning technology, communication technology and other high-tech Internet Technology technology has also been applied to many industries in. The birth of the technology is considered to be a great revolution in the history of the global information industry, has been the global attention and attention, each country is currently actively committed to the application of Internet Technology technology research. In the 21st century, which is the theme of intelligence, energy conservation and informationization, it is undoubtedly the inevitable trend and requirement of the development of the Internet in the fields of transportation, electricity and architecture.

\section{The Connotation and Characteristics of Internet Technology}

Internet Technology is mainly through a variety of information sensing equipment to collect any need to monitor control, connection and interaction of objects and processes, it collects sound, electricity, heat, chemistry, biology and other required information, and with the Internet Combined to become a huge network. Its main purpose is to achieve people and things, as well as objects and people and other items can be connected with the network to facilitate people to identify, manage and control and so on.

The Internet Technology itself is a network, but it is built on an extensive network on the Internet. But it lacks independent features that are different from the Internet. First of all, it incorporates a large number of perceptual technologies, and its information is mainly through the distribution of the network at the end of the various types of sensor devices, these sensors for the Internet Technology is equivalent to its "eye", "ear". Second, the Internet Technology in the "material" through a variety of wired or wireless communication connected to the Internet, in order to achieve the information upload or instructions issued, and then to achieve integration with the Internet. Third, the Internet Technology not only through the sensor equipment to exchange all kinds of information, its own information has the ability to deal with, through this ability to achieve the intelligent control of the object. So, the Internet Technology is a combination of Internet technology and sensor technology and can only deal with the formation of a large technology such as network technology.

\section{The Key Technology of Internet Technology}

M2M technology, sensor network technology and radio frequency identification (RFID) technology, network communication technology is the key technology of things.

M2M technology. M2M technology through the realization of the machine and the machine, people, people and the communication between the machine, and the operator to share the machine 
equipment, application processing and acquired information system to provide information. M2M technology provides an excellent means of transmitting data, enabling it to make wireless connections between systems, between remote devices, and between individuals in real time.

M2M products mainly consist of three parts:

The wireless terminal: not a laptop or mobile phone, but a special industry application terminal; (3) transmission channel: from the following: (1) the industry application center: to monitor the scattered industry terminals, terminal convergence data is the convergence point; Client-to-wireless channel between the industry application centers.

Increasing the number of M2M end devices connected to the object, the number will be more than the number of computers and people. In the software management platform, through the M2M management software, you can achieve the effective management of assets and end devices, control. In the hardware manufacturing of things, the machine has the networking or communication capacity of the components is M2M hardware, M2M hardware can extract the information can also be obtained from the machine equipment and data transmission to the communications network hardware manufacturers, different applications. The environment's mobile information processing can be achieved through different wireless M2M hardware products. A number of industries have been involved in M2M technology, M2M technology has been able to achieve "let the equipment speak", the machine is no longer an information island, which can be achieved on the effective management of assets and equipment and monitoring, through improvement Services, optimize the cost of configuration to promote social information to a more environmentally friendly, efficient, energy saving, security direction.

Sensor network technology: large-scale wireless sensor network technology, sensor and its intelligent processing technology is the combination of sensor network technology. Because it is a detection device, the sensor can feel the measured information, and can detect the information, according to a certain law of conversion into electrical signals or other forms of information output to meet the information storage, transmission, display, Record, processing and other requirements. To achieve automatic control and automatic detection of the first part is the sensor, in practice and the sensor is equivalent to the "sensory organs." New technology of low power consumption, miniaturization, mobile, low cost a bit to meet the " The wireless sensor network can meet the above needs under the premise of providing automatic repair function and automatic network of mesh network, so that the wireless network has the initial wisdom of the function. With the arrival of the new technological revolution, the world has entered a new era of information technology. In practical applications, the first thing to solve is how to obtain accurate and credible information, and in the use of information in the process, the sensor has a very prominent position, it is because the sensor is to obtain the means of production and natural information means and the main way.

Radio frequency identification (RFID) technology: Usually, when a particular information reader through the electronic tag with the items, the reader to activate the label, and to the reader and information processing system to send the information in the label, so as to complete the automatic collection of information. A typical RFID system is composed of readers, RFID tags and information processing systems. The information processing system shall bear the corresponding information processing and control work according to the demand. Since each RFID tag has a unique identifier, if its data format is a lot of incompatible, in the case of closed-loop, the impact on the enterprise is not great.

Network communication technology: Internet Technology data is through the sensor network communication technology to provide transmission channel. At present, the focus of Internet Technology research is how to enhance the existing network, to meet the needs of things networking. Network communication technology can be divided into two categories: wide area network communication technology and close communication technology. In the wide area network communication, satellite communication technology, 2G / 3G mobile communication technology to achieve the remote transmission of information, you can assign IP address for each sensor to create the possibility of the development of the sensor can also create a good basis for network conditions. In terms of short-range communication, 802.15.4 specification is developed by IEEE for low-speed 
short-range communication media intervention control layer and physical layer specification, IEEE802.15.4 as the representative of the short-range communication technology is the mainstream technology, work in industry scientific medical band, license-free 2.4 Ghz ISM band can be used worldwide.

\section{Application of Internet Technology}

Industrial areas. As one of the three key technologies of the Internet Technology, RFID technology is a huge change in the supply chain field because it has the advantage of recognizing distance far, fast and easy to damage, and large bar codes that cannot be compared. Because of the advanced nature of RFID technology, it can also be used in the field of intelligent transportation, to achieve intelligent container management.

Agriculture. Internet Technology can be achieved remote control and real-time collection function, which makes the Internet Technology in the application of agriculture is very extensive. The use of Internet Technology can be transmitted through the wireless signal transceiver module, you can automatically turn on or off the designated equipment, adjust the temperature and humidity and other environmental conditions, in order to achieve the greenhouse temperature and humidity remote control. But also real-time collection of greenhouse temperature and humidity signals and light, soil temperature, $\mathrm{CO} 2$ concentration and other environmental parameters, and these information at any time for the comprehensive agricultural ecological information automatic monitoring, environmental control and intelligent management to provide a scientific basis. Also in the sale of products, you can use a mature Internet Technology sensing technology, eco-agricultural base and consumers to build a network between the sales platforms. So that consumers can through real-time network video to understand the whole process of planting agricultural and sideline products, the product more confidence. Fast, low cost, reliable perception of process information is the basis of the Internet Technology application.

Transportation. The use of advanced Internet Technology to the entire land, sea, aviation, pipeline traffic management system, so as to establish a wide range of all-round role in the efficient, convenient, safe, environmentally friendly, comfortable, real-time, accurate integrated transport Management system.

Aquaculture areas. In the farm to implement the construction of things, for some aquaculture animal aquaculture quality of online monitoring and control, aerobic facilities, such as automatic control of the project. In the water temperature, ph value, dissolved oxygen, ammonia, hydrogen sulfide, nitrite and other key indicators, through the monitoring point of real-time measurement data, wireless transmission, to achieve automatic control or transmission of video information, managers in the computer Screen and mobile phone for real-time monitoring and manual control, making the development of aquaculture industry has become more smooth sailing.

Intelligent home appliances field. The intelligent home appliance product integrates the automation control system, the computer network system and the network communication technology, and realizes the automation of the home equipment through the Internet Technology, through the China Telecom's broadband, fixed line and 3G network can realize the remote control of the home equipment the Internet air conditioning is in accordance with the agreed agreement, through the sensor equipment, directly to the air conditioning terminal for information exchange and communication, in order to achieve intelligent identification, tracking, monitoring and management of a network air conditioning. Internet water heaters use radio frequency automatic identification technology, set up by the user, smart phones, wireless routing, water heater equipment, cloud server consisting of things networking platform, allowing users to understand the working state of the water heater through the smart phone, and can operate it. 


\section{The Development Trend of Internet Technology}

Things are very broad prospects, it will greatly change our current way of life. First, the application of Internet Technology can improve economic efficiency and cost savings. Secondly, the development of Internet Technology can drive the development of many related industries and provide sustainable power for the global economy. In addition, the application of Internet Technology can greatly facilitate our life. In the world of things, each object has a certain degree of intelligence and you can automatically complete some of the previous needs of human intervention to complete the matter. Things are good, but to build a truly efficient and practical Internet Technology, there are two factors essential. First, the scale, that access to the network of objects must reach a certain size, only with the scale, the role of intelligence can really play out. For example, if there are millions of cars on a city's road, if we only have ten thousand cars connected to the network, we cannot have a comprehensive understanding of the traffic in the city, nor can we build a smart traffic System; followed by mobility, the object is usually in motion, to ensure that the object in the state of motion, and even high-speed movement can be carried out at any time data exchange, which requires the establishment of supporting information on the highway, especially large capacity Mobile interconnection channel. EPOSS in the "Internet Technology 2020" report analysis and prediction, the future development of the Internet Technology will go through four stages, before 2010, radio frequency identification technology will be widely used in logistics, retail and pharmaceutical fields, 2010-2015 Internet, 2015 -2020 objects into semi-intelligent, after 2020, the object into the full intelligence. At present, the first phase of the function has been achieved at home and abroad, is to the second stage of the goal.

\section{Conclusion}

The rise of the Internet Technology is another wave of information industry and the Internet Technology is widely regarded as the post-financial crisis era to drive the world's economic growth and the new engine of the founder in the future. The development of the Internet Technology is not a step in place, it is designed a lot of content. In China only rely on the government and scientific research units, business units and the social industry work together, so that the Internet itself can be found the great potential which reflects its due value.

\section{Acknowledgment}

The paper is supported by the project of Research on the application of virtual machine dynamic allocation technology in IaaS , 2016 Scientific Research Program Funded By Shaanxi provincial Education Departmen (No.16JK2176) .

\section{References}

[1] B. N. Ma, Talking about the Internet Technology and its application, J. Computer Knowledge and Technology. 44 (2010) 146-147.

[2] H.X.Zeng, Analysis of the application and development of things, J. Science and Technology Information 18(2005) 55-57.

[3] J.H.Xue, Internet Technology and Information Development, J. Association for Science and Technology. 32(2012) 189-190.

[4] Y.J.Qi, Research on Key Technology of Production Execution System Based on RFID Technology, J. Journal of Ocean University of China, 4(2007) 38-40.

[5] Y.M.Tian, Review of Research on Internet Technology, J. Journal of Electronics Measurement \& Instrumentation 9(2013) 142-150. 